\title{
Making movies of stars: VLBA monitoring of the SiO masers around the Mira variable TX Cam
}

\author{
P. J. Diamond and A. J. Kemball \\ NRAO, PO Box 0, Socorro, NM, 87801, USA
}

\begin{abstract}
We have produced a movie of the time evolution of the $v=1$, $\mathrm{J}=1-043 \mathrm{GHz} \mathrm{SiO}$ masers in the inner circumstellar envelope of the isolated Mira variable TX Cam. Our observations, taken every two weeks with the VLBA, currently cover about $60 \%$ of the stellar cycle from $\phi \sim$ $0.6 \rightarrow \phi \sim 1.2$. They show that, in contrast to previous observations and theoretical models, the gas in which the masers are embedded is undergoing expansion during this period with a typical velocity of $3.65 \pm$ $0.15 \mathrm{~km} / \mathrm{s}$. Some isolated features are seen to start falling back towards the star at around $\phi \sim 1.0$. We suggest that the $5 \rightarrow 10 \mathrm{G}$ magnetic fields we have previously detected at the position of the $\mathrm{SiO}$ masers may explain the departure from the expected behaviour.
\end{abstract}

\section{Introduction}

Circumstellar envelopes can be studied in many ways as the numerous papers presented at this meeting have shown. However most observational studies deal with data taken at a single epoch or coarsely sampled data studying the largescale properties of the envelopes or particular chemical species. Theoretical studies based on the observations attempt to reproduce the gross properties of the envelopes and the underlying stellar structure and have only recently attempted to describe the dynamics involved. More detailed theoretical work has been hampered by the lack of high-resolution, high time-sampled data that reveal the true structure and evolution of the circumstellar envelopes.

With the advent of NRAO's Very Long Baseline Array (VLBA) we now have a sensitive, 10-station interferometer, observing year round with a spatial resolution $\sim 50-100$ times that of the Hubble Space Telescope. One of the stated goals of the VLBA is to enable frequent time monitoring of radio sources. Observations of molecular masers in the circumstellar envelopes of late-type stars are well suited to such an instrument.

Habing (1996) has produced an excellent review on the subject of circumstellar envelopes and describes in some detail the location and structure of $\mathrm{OH}$, $\mathrm{H}_{2} \mathrm{O}$ and $\mathrm{SiO}$ masers. Masers are detectable by VLBI techniques since they are compact high brightness temperature objects. They are thus very useful probes of the structure and dynamics of the clouds in which they reside. Habing (1996) sums up the available literature on $\mathrm{SiO}$ masers which show that they lie close to the stellar photosphere inside the dust condensation radius and, in most stars, form a beautiful ring-like structure (Diamond et al. 1994). One obvious 
conclusion, based on the observed rings, is that the $\mathrm{SiO}$ masers are tangentially amplified unlike the radial amplification seen in $\mathrm{OH}$ masers in the same stars. The $\mathrm{SiO}$ masers also exhibit strong linear polarization with a highly ordered position angle structure primarily tangential to the envelope (Kemball \& Diamond 1997).

Little is known about the variability of $\mathrm{SiO}$ maser structure. The current state of knowledge has been gleaned principally from single-dish observations (e.g. Cho et al. 1996; Pijpers et al. 1994). There appears to be little connectivity of spectra and spectral features from cycle to cycle of the underlying stellar pulsation. From day to day changes appear minor and are mainly confined to simple flux density variability. However, within a stellar cycle there do appear to be characteristics of the variability that are common to most stars:

- the $\mathrm{SiO}$ maser flux density varies in the same manner as the optical flux but with a phase lag $(\Delta \phi \sim 0.2)$;

- the brightness contrast between minimum and maximum SiO maser flux density varies from $5 \rightarrow 100$ depending on the star;

- $86 \mathrm{GHz} \mathrm{SiO}$ masers $(\mathrm{v}=1, \mathrm{~J}=2-1)$ are brighter for longer than $43 \mathrm{GHz}$ masers $(\mathrm{v}=1, \mathrm{~J}=1-0)$;

- from $\phi \sim 0.2 \rightarrow 0.8$ the average velocity of the $43 \mathrm{GHz} \mathrm{SiO}$ spectrum may be red-shifted with respect to the stellar velocity implying that the gas is infalling (Cho et al. 1996).

Boboltz et al. (1997) showed, through VLBA observations at three epochs, that the $\mathrm{SiO}$ masers around the symbiotic Mira $\mathrm{R}$ Aquarii were falling back towards the star with an average velocity of $4.2 \pm 0.9 \mathrm{~km} / \mathrm{s}$ over a phase range of $0.78 \rightarrow 1.04$. This is in agreement with the statistical single-dish results of Cho et al. (1996) mentioned above and the theoretical models of Bowen (1988). One result from the Boboltz et al. (1997) observations was that in order to adequately follow individual maser components with time the optimum VLBI observing interval was 2 weeks. In this contribution we present preliminary results from such a monitoring program of the $43 \mathrm{GHz}(\mathrm{v}=1, \mathrm{~J}=1-0) \mathrm{SiO}$ masers towards the isolated Mira variable TX Cam which has a period of 557 days and lies at a distance of 317 pc. Previous observations of the masers around TX Cam (Diamond et al. 1994; Kemball \& Diamond 1997) have shown them to lie in a well-defined ring structure with a typical diameter of 30 milliarcsec and with a polarization structure which shows electric vectors lying predominantly tangential to the ring.

\section{Observations}

Multiple epoch VLBA observations at two week intervals of the $\mathrm{SiO}$ maser in TX Cam began in May 1997 and are still running. We hope to obtain 50 epochs to cover 1.25 cycles of the Mira's pulsation. In this paper we report on the preliminary results from the first 21 epochs. The AAVSO light curve of TX Cam is shown in Fig. 1 with the time of each VLBA observation marked with 


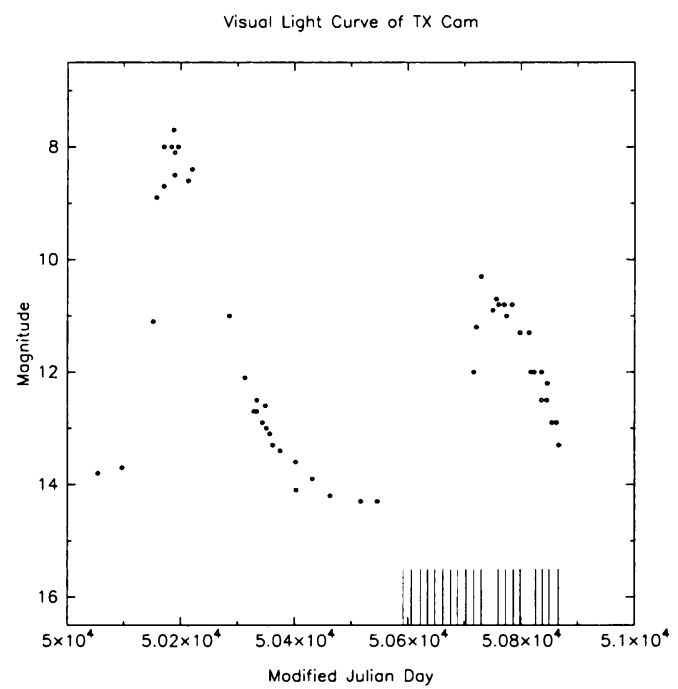

Figure 1. The light curve of TX Cam (courtesy of AAVSO). The vertical lines at the bottom of the plot represent the times of VLBA observations used to produce the movie.

a vertical line. The phase range covered by the current movie is from $\phi \sim 0.6$ to $\phi \sim 1.2$, that is similar to, but larger than, the phase range covered by the Boboltz et al. (1997) observations of R Aquarii mentioned above.

At each epoch we obtain 6 hours of data on TX Cam and correlate the data at the VLBA correlator in Socorro, NM producing 128 channel, 4 Stokes parameter data for all available baselines. Most observations include a single antenna of the VLA. In order to handle the enormous amount of data generated we have developed an automatic data calibration and imaging procedure within the AIPS package. This has proven highly effective and has enabled us to keep up with the influx of data.

Work is continuing on semi-automatic methods of image analysis which will result in our final data product: a large list of component positions, fluxes and polarization properties. However, it is possible to visualize the structural changes in the $\mathrm{SiO}$ maser emission by making a movie. This is constructed by first taking the Stokes I image cube of each epoch and running through the cube in velocity space, extracting the maximum value of each pixel and forming a composite image that represents the total structure well. The resultant images at each epoch have to be registered in some manner since we do not have absolute position measurements of the required accuracy ( $\mu$ arcsec level); this is done by assuming that there are no major changes between epochs and then cross-correlating the images and performing pixel level shifts to maximize the correlation. Such a method works well and justifies our desire to observe every two weeks. The final step is to interpolate across the occasional gap that occurs in our sequence of observations (due to operational limitations, bad weather etc.) and then form a new cube which now has time as the third axis. 

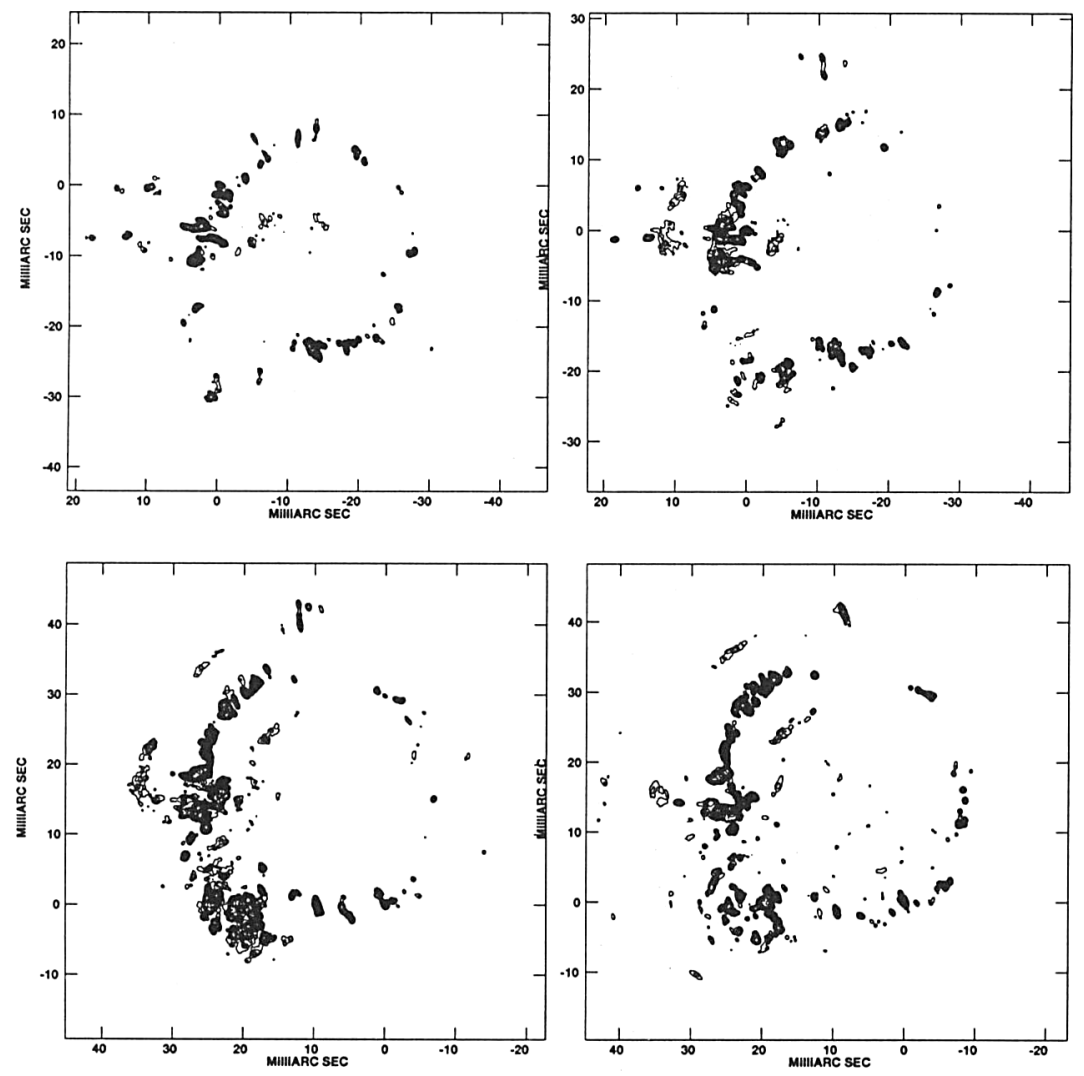

Figure 2. Four frames of the VLBA movie of TX Cam. The dates of observation are: top left - 24 May 1997; top right - 16 Aug 1997; bottom left - 5 Dec 1997; bottom right - 22 Feb 1998

\section{The movie}

Fig. 2 shows four frames from the movie shown during the conference. The principal result is that the dominant dynamical mode is one of expansion. The first epoch $(24 / 5 / 97)$ shows a largely complete, almost circular ring, with an average diameter of 31 milliarcsec $(9.8$ a.u.). The final epoch $(22 / 2 / 98)$ shows a structure that has transformed into an ellipse with an EW diameter of 34 milliarsec (10.8 a.u.) and a NS diameter of 36 milliarcsec (11.4 a.u.). The changes are highly significant being of order ten times larger than the VLBA's beamsize at $7 \mathrm{~mm}$ and correspond to a $10 \%$ increase in size in the EW direction and $20 \%$ in the NS.

We can follow the motion of individual features. Gaussian fitting of maser components at each epoch will produce proper motion diagrams. Fig. 3 shows the data for one such feature in the NE quadrant of the ring. A fit to the data points shows that, in the 6 month period before the feature faded, it maintained 
a constant outflow velocity of $3.65 \pm 0.15 \mathrm{~km} / \mathrm{s}$. This velocity is consistent with the width of the $\mathrm{SiO}$ maser spectrum.

The large complex of features that lies in the SE portion of the ring increased in flux during the observation period and shows evidence of peculiar motions with features sliding in non-radial directions. It is possible that this complex is indicative of non-symmetric mass-loss.

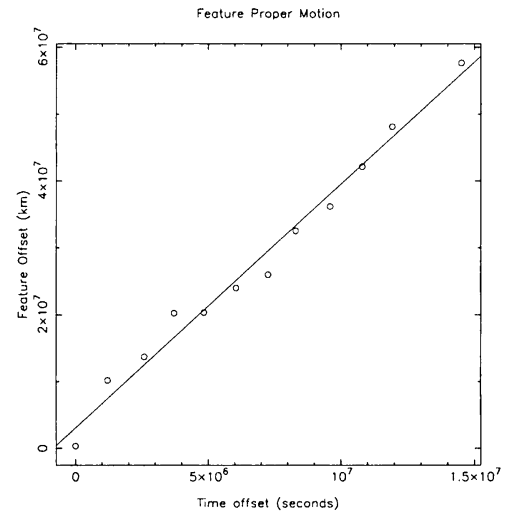

Figure 3. The proper motion of a feature in the NE quadrant of the TX Cam maser ring. The errors bars for each feature are smaller than the circles on the plot. The feature fades from view after 6 months. The straight line is a fit to the data which results in a feature proper motion of $3.65 \pm 0.15 \mathrm{~km} / \mathrm{s}$.

\section{Discussion}

The continual expansion of the maser ring from $\phi \sim 0.6 \rightarrow \phi \sim 1.2$ is in direct contrast to the contraction observed in R Aquarii (Boboltz et al. 1997) and implied by the statistical single-dish observations of Cho et al. (1996). It also contradicts the theoretical model proposed by Bowen (1988) and recently expanded upon by Gray et al. (1998). The current theoretical understanding of the variations of the $\mathrm{SiO}$ masers is summed up by Gray et al. (1998) who state that flux and line profiles changes are expected as the masing objects move within a region with changing density, temperature and radiation fields. An abrupt change is expected once per stellar cycle due to the passage of the outward-moving shock front. Based on various observations of the $\mathrm{SiO}$ masers in R Aquarii Gray et al. (1998) suggest that the shock wave moves through the photosphere at a visual phase of $\phi \sim 0.7$ and that one would then expect to see contraction due to the star's gravitational field and flux increase of the maser emission from $\phi \sim 0.8 \rightarrow \sim 0.4$.

In the case of TX Cam we do observe a flux increase throughout the period of observation with a levelling off towards the end. However, as stated above the masers appear to be generally expanding at a more or less constant velocity. A few isolated maser features appear at $\phi=1.0$ and begin to fall back towards the star but not along radial paths. 
A major question is: what mechanism supports the observed continual expansion? The models of Bowen (1988) suggest quite strongly that after the impulse imparted by the passage of the shock wave gravity should eventually slow and then pull back the expanding gas. $\mathrm{R}$ Aquarii seems to follow this model yet TX Cam does not. We suggest that the stellar magnetic fields may play a crucial role in the dynamics of the inner envelopes.

Using circular polarization measurements Kemball \& Diamond (1997) estimated the magnetic field strength in the vicinity of the $\mathrm{SiO}$ masers around TX Cam to be $B \sec \Theta \sim 5-10 \mathrm{G}$, where $\Theta$ is the angle between the line of sight and the magnetic field. As was pointed out by Barvainis et al. (1987) the ratio of magnetic to thermal energies in the gas of the circumstellar envelope is $>1$ for magnetic fields of this strength. Even for field strengths of $\sim 0.1 \mathrm{G}$ the overall dynamics of the gas will be strongly influenced by the magnetic fields. Future analysis of the polarization structure visible in our data should provide us with crucial information on the role of magnetic fields in the circumstellar environment.

Our VLBI monitoring data of SiO masers around TX Cam provides us with a wealth of data on the structure, motions, time evolution, mass-loss process, magnetic field structure, conditions and overall dynamics of the inner regions of the circumstellar envelope. We have only scratched the surface of this immense dataset in producing the movie.

Acknowledgments. The National Radio Astronomy Observatory is a facility of the National Science Foundation operated under cooperative agreement by Associated Universities, Inc. We acknowledge with thanks, data from the AAVSO International Database, based on observations submitted to the AAVSO by variable star observers worldwide.

\section{References}

Barvainis R., McIntosh G., Predmore C.R., 1987, Nature 329, 613

Boboltz D.A., Diamond P.J., Kemball A.J., 1997, ApJ 487, 147

Bowen G.H., 1988, ApJ 329, 299

Cho S., Kaifu N., Ukita N., 1996, AJ 111, 1987

Diamond P.J., Kemball A.J., Junor W., Zensus J.A., Benson J., Dhawan V., 1994, ApJ 430, 61

Gray M.D., Ivison R.J., Humphreys E.M.L., Yates J.A., 1998, MNRAS 295, 970

Habing H.J., 1996, Astron. Astroph. Rev. 7, 97

Kemball A.J., Diamond P.J., 1997, ApJ 481, 111

Pijpers F.P., Pardo J.R., Bujarrabal V., 1994, A\&A 286, 501 